\title{
Improved ADRC of Airborne Electro-optical Stabilized Platform
}

\author{
Qixuan Zhu ${ }^{1,2, *}$, Honggang Zhang ${ }^{2}$ and Junke Gao ${ }^{2}$ \\ ${ }^{1}$ Science and Technology on Electro-Optical Control Laboratory, Luoyang 471000, China \\ ${ }^{2}$ Luoyang Institute of Electro-Optical Equipment, AVIC, Luoyang 471000, China \\ ${ }^{*}$ Corresponding author
}

\begin{abstract}
This paper proposed a method for perameter setting of traditional nonlinear extended state observer using Back-Propagation neural network .This method simplified the complicated nonlinear extended state observer parameter setting process. This paper simulated the active disturbance-rejection control system combining Back-Propagation neural network. The simulation results show that this method can significantly enhance the disturbance isolation of the airborne optoelectronic stabilized platform, and the control effect is obviously superior to the traditional active disturbance-rejection control method, which is of great significance to improve the axis stability accuracy of the airborne optoelectronic stabilized platform.
\end{abstract}

Keywords-component; optoelectronic stabilized platform; extended state observer; active disturbance-rejection control; backpropagation neural network; disturbance compensation

\section{INTRODUCTION}

As the "eye" of an aircraft, the optoelectronic stabilized platform has the functions of capturing, tracking and aiming at the target. Pointing detectors will be interfered by various disturbances during the flight, which will affect the accuracy of line of sight stabilization. To improve the disturbance isolation can improve the accuracy of line of sight(LOS) stabilization, and makes the image of the optoelectronic stabilized platform clearer and the information acquisition more accurate[1,2].

The traditional control strategy achieves the effect of isolating disturbance by increasing the open-loop cutoff frequency of the system. However, because the open-loop cutoff frequency of the system is strictly limited by the mechanical resonance frequency, the gain of the system in the disturbance frequency band can't be further improved[3]. This limits the improvement in stability accuracy.

Active Disturbance-rejection Control (ADRC) uses disturbance compensation control to suppress disturbance effects. Extended state observer(ESO) is the core of ADRC, the "total disturbance" in the system is observed and compensated by ESO in real time, which greatly improves the disturbance isolation and improves the stability accuracy of the optoelectronic stabilized platform [4,5].

The parameter tuning of ESO is very important to the performance of the observer, Dr. Gao Zhiqiang from Cleveland State University proposed a single parameter [6] design method based on bandwidth concept. These methods have better robustness and can be adapted to various models after adjustment.
The intelligent algorithm with adaptive parameters tuning can improve system performance while maintaining good robustness of the system. Some literatures have tried to adjust parameter of ADRC using the method similar to the method of BP neural network tuning PID [7]. However, most of them only focus on two parameters of Non-linear state error feedback (NLSEF) $[8,9]$. In fact, the parameter tuning method of the ESO is more complex. At present, the empirical formula is mainly used to adjust it because the accurate model of the system is difficult to obtain. This method is not only timeconsuming, but also difficult to determine the optimal parameters. Therefore, the adaptive adjustment of BP neural network is of great significance for improving the performance of ESO.

\section{THE THEORY OF ADRC}

ADRC was first proposed in the late 1980s by Han Jingqing a researcher at the Chinese Academy of Sciences. Its core idea is "active disturbance rejection". All the external disturbances and the "uncertain dynamics" of the system are called "total disturbance" and the "total disturbance" is estimated in real time by the ESO, to realize the direct feed forward compensation control for disturbance.

ADRC includes three parts: Tracking Differentiator (TD), ESO and NLSEF control law.

\section{A. Tracking Differentiator}

The main function of the TD is to arrange the appropriate transition process according to the control target and the object's endurance, resolving the contradiction between rapidity and overshoot of the system. The steepest TD shown in equation (1) is used in this paper.

$$
\left\{\begin{array}{l}
x_{1}(k+1)=x_{1}(k)+h x_{2} \\
x_{2}(k+1)=x_{2}(k)+h f h \\
f h=f h a n\left(x_{1}(k)-v(k), x_{2}(k), r, h\right)
\end{array}\right.
$$

in this equation, $x_{1}$ is the tracking value of instruction, and $x_{2}$ is the tracking value of instruction differential. fhan $\left(x_{1}(k)\right.$ $\left.v(k), x_{2}, r, h\right)$ is the system's fastest control synthesis function, and its specific algorithm as shown in the formula (2): 


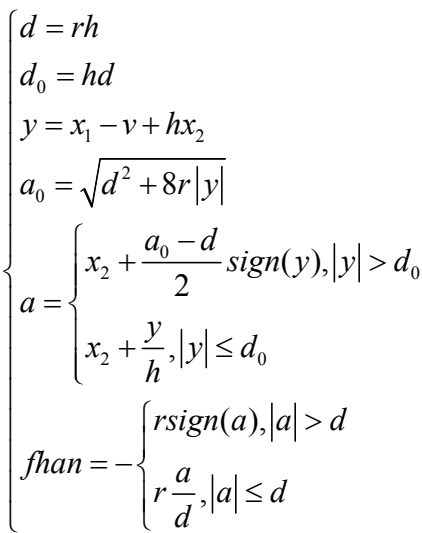

In this equation, $r$ is the speed factor, and the greater the value of $r$, the faster the tracking speed, $h$ is the sampling time, and the rest are intermediate variables.

\section{B. Extended State Observer}

In this paper, the two order nonlinear controlled system with disturbance is considered as the object of study. The principle of the ESO is discussed, and the three order discrete extended state observer is constructed, such as equation (3):

$$
\left\{\begin{array}{l}
e_{1}(k)=y(k)-z_{1}(k) \\
z_{1}(k+1)=z_{1}(k)+h\left(z_{2}(k)+\beta_{1} e_{1}(k)\right) \\
z_{2}(k+1)=z_{2}(k)+h\left(z_{3}(k)+\beta_{2} f a l\left(e_{1}, \alpha_{1}, \delta_{1}\right)+b u\right) \\
z_{3}(k+1)=z_{3}(k)+h \beta_{3} \text { fal }\left(e_{1}, \alpha_{2}, \delta_{2}\right)
\end{array}\right.
$$

In this equation, $\beta_{i}$ are the parameters to be adjusted, and the BP neural network method is used to adjust the three parameters. fal $\left(\mathrm{e}_{\mathrm{i}}, \alpha_{\mathrm{i}}, \delta_{\mathrm{i}}\right)$ is a nonlinear function of the ESO, which has been summed up by Han Jingqing researcher for many years in the study of ADRC:

$$
\operatorname{fal}\left(e_{1}, \alpha_{i}, \delta_{i}\right)=\left\{\begin{array}{l}
\frac{e_{1}}{\delta^{1-a_{i}}}, \quad\left|e_{1}\right| \leq \delta_{i} \\
\left|e_{1}\right|^{a_{i}} \operatorname{sign}\left(e_{1}\right), \quad\left|e_{1}\right|>\delta_{i}
\end{array}\right.
$$

By adjusting the appropriate $\alpha_{\mathrm{i}}$ and $\delta$ can get proper nonlinear functions, nonlinear ADRC has better ability of disturbance estimation and compensation, and parameters adjustment of nolinear ADRC is more complex.

\section{Nolinear Control Law}

In this paper, the control law is generated by the combination of the instruction signal and the observation signal, which is similar to the PD control. The nonlinear control law is used as shown in equation (5):

$$
u_{0}=k_{1} \bullet \operatorname{fal}\left(e_{1}, \alpha_{3}, \delta_{3}\right)+k_{2} \cdot \operatorname{fal}\left(e_{2}, \alpha_{4}, \delta_{4}\right)
$$

In this equation, $\mathrm{k}_{1}$ and $\mathrm{k}_{1}$ are the parameters to be adjusted [10], and they are also adjusted by BP neural network in this paper.

\section{ADRC PARAMETER TUNING METHOD BASED ON BP NEURAL NETWORKG}

\section{A. Structure of ADRC Parameters Tuning Method Based on BP Neural Network}

Parameter tuning of ESO is an important part of ADRC design. Since the ADRC does not depend on the exact model of the system, the method of ESO parameters adjustment often adopt the empirical formula. The real-time tuning of ESO parameters based on BP neural network, ESO can be changed with the change of system disturbance, adjust the parameter self-adaptively. Using this method is equivalent to doing the automatic adjustment of the system identification, which can improve the ADRC control system robustness and improve the accuracy of the measurements of ESO, thus improving the stability accuracy of the system. Fig. 1 is a block diagram of ADRC Parameter Tuning Method based on BP Neural Network

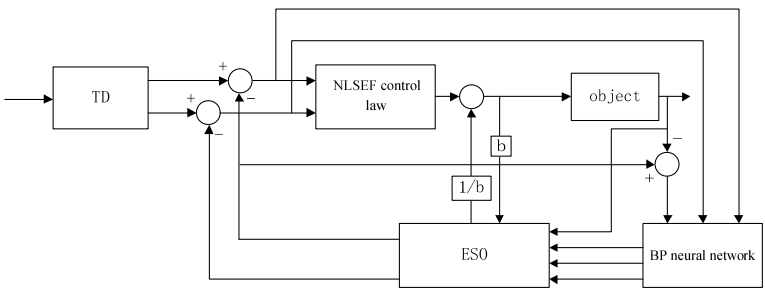

FIGURE I. BLOCK DIAGRAM OF ADRC PARAMETER TUNING METHOD BASED ON BP NEURAL NETWORK.

In this paper, three layer BP neural network is used to adjust ESO parameters based on BP neural network, the input of the neural network should select several signals which are most closely related to the observation accuracy of ESO. After experiments, the 4 input nodes of the neural network are chosen, which are the signal error $E 1$, the signal differential error E2, the observation error "error", the three signals and the bias value 1 , the 3 output layer nodes are the ESO parameters $\beta_{1}, \beta_{2}, \beta_{3}$, and the 5 hidden layer nodes are selected by combining the specific controlled object and the trial. Then the internal structure of the BP neural network is shown as shown.

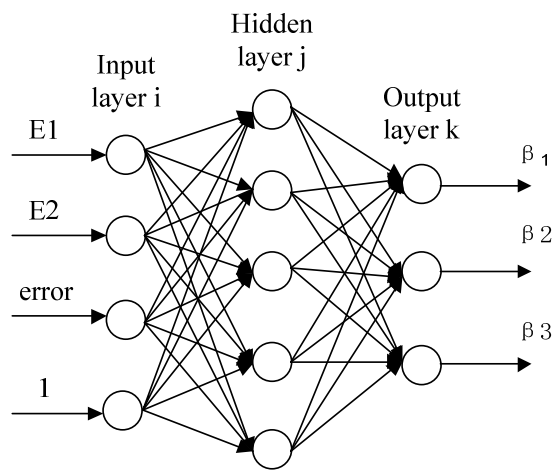

FIGURE II. INTERNAL STRUCTURE OF THE BP NEURAL NETWORK.

Because the activation function of the output layer of the neural network often choose sigmoid function, its characteristic is its output value is non-negative and is less than or equal to 1 , 
while the ESO parameters are not within this range, so ESO parameters tuning using BP neural network should adjust the output values of the BP neural network, then use the adjusted values as ESO parameters. Because the BP neural network Corrects weight coefficients according to the gradient descent method, the disadvantage of this method is that if the output value and ideal output deviation is too large it is easy to diverge therefore need to select the suitable coefficient of the output layer, make the neural network better convergence.

\section{B. A Brief Introduction of BP Neural Network for Tuning ESO Parameters}

The output of the neural network input layer as defined above is:

$$
\mathrm{O}_{i}^{i n}=x(i), i=1,2,3,4
$$

The inputs and outputs of the hidden layer are:

$$
\left\{\begin{array}{l}
N e t_{j}^{i m}(k)=\sum_{i=1}^{4} w_{j i}^{i m} O_{i}^{i n} \\
O_{j}^{i m}(k)=f\left(\operatorname{Net}_{j}^{i m}(k)\right)
\end{array}, i=1,2,3,4,5\right.
$$

The activation function of hidden layer neurons is hyperbolic tangent function:

$$
f(x)=\frac{e^{x}-e^{-x}}{e^{x}+e^{-x}}
$$

The inputs and outputs of the output layer are:

$$
\left\{\begin{array}{l}
\operatorname{Net}_{k}^{\text {out }}(k)=\sum_{i=1}^{4} w_{k j}^{\text {out }} O_{j}^{\text {im }} \\
O_{k}^{\text {out }}(k)=g\left(\operatorname{Net}_{k}^{\text {out }}(k)\right) \times \mathrm{A}_{k}
\end{array}, k=1,2,3\right.
$$

The 3 output nodes of the output layer correspond to $3 \mathrm{ESO}$ parameters $\beta_{1}, \beta_{2}, \beta_{3}$ respectively, and $A_{k}$ is the output layer coefficient, and the output layer neuron activation function uses the non-negative sigmoid function:

$$
g(x)=\frac{e^{x}}{e^{x}+e^{-x}}
$$

Defined the cost function of the system is:

$$
\varepsilon(k)=\frac{1}{2}(\operatorname{rin}(k)-\operatorname{yout}(k))^{2}
$$

The application of back-propagation algorithm of BP neural network is very proven. So we don't give too much detail.

\section{Output Layer Coefficient Selection Method}

This paper proposes a method. Multiply the output layer activation function outputs and the corresponding output layer coefficients trained by the BP neural network, and then use it as the ESO parameter:

Firstly, the single parametric design method based on bandwidth concept which is proposed by Dr.Gao Zhiqiang is used to select the base values of a set of initial coefficients $[3 \omega$ $\left.3 \omega^{2}, \omega^{3}\right]^{[6]}$. $\omega$ is the parameter related to system bandwidth, and the greater the value of $\omega$, the higher the bandwidth of the ESO

Because output value of the sigmoid function is nonnegative and is less than or equal to 1 . Therefore, the initial coefficient should be multiplied by a factor $\mathrm{k}$ greater than 1 . Then, after the BP neural network is trained in the system, a set of parameters $\left[\mathrm{A}_{1}, \mathrm{~A}_{2}, \mathrm{~A}_{3}\right]$ of convergence is obtained. With this group of parameters multiplied by $\mathrm{K}$ as the initial factor, the training is performed again, and the value of $\mathrm{X}$ can be adjusted in training until the convergence speed of training is faster.

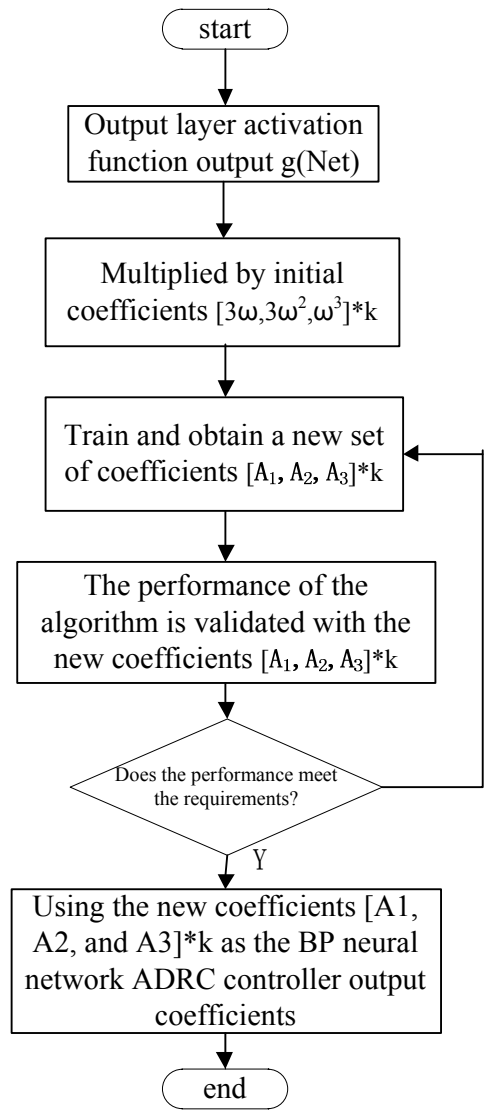

FIGURE III. INTERNAL STRUCTURE OF THE BP NEURAL NETWORK.

\section{SimUlATION ANALYSIS}

\section{A. Photoelectric Stabilized Platform Configuration}

Photoelectric stabilized platform servo control system uses gyroscope, resolver encoder, image tracker etc. as the feedback element, the controller input signal processing and feedback signal and drives the motor to control the load platform. The controller processes the input signal, the feedback signal and drives the motor to control the load of the platform. The controller adjusts PWM wave signal to control the motor, so as 
to adjust the direction of LOS. Gyro sense the LOS speed in inertial space, the encoder sense the angle of the mirror relative to the carrier, the image tracker output target miss distance. The schematic diagram of the working principle is shown in the following figure.

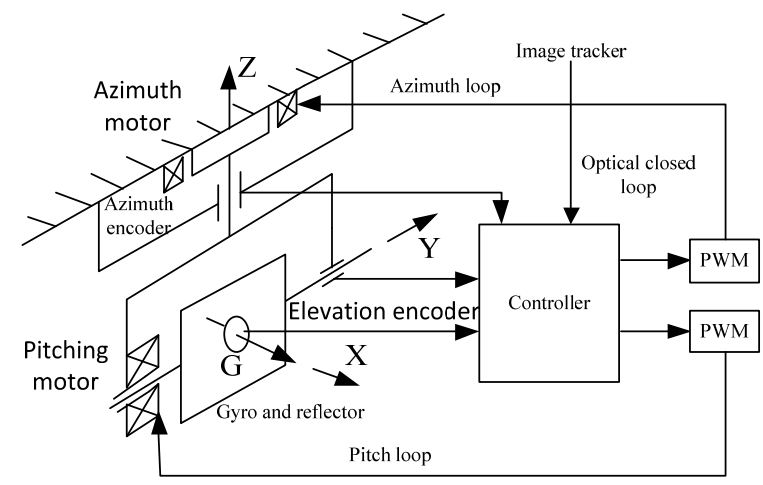

FIGURE IV. DIAGRAM OF A DOUBLE-AXIS STABILIZED PLATFORM OPERATING PRINCIPLE

The flow chart of the servo system of the single axis stabilization loop is shown in the following figure:

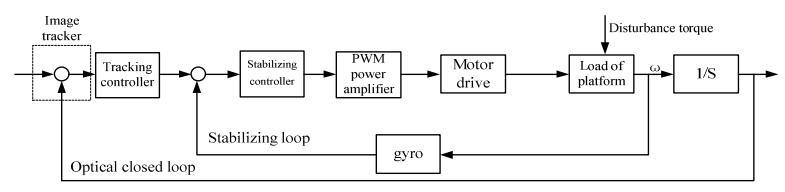

FIGURE V. DIAGRAM OF A SINGLE-AXIS STABILIZATION LOOP

Firstly, determine the controlled object model used for simulation. According to the parameters of the common airborne photoelectric stabilized platform, the model of the system is established:

$$
\frac{1.25}{0.001034 s^{2}+1.804 s+1.5625}
$$

The disturbance model of the system is established according to the common disturbance parameters in the stability test.

\section{B. Disturbance Analysis and Modeling}

When the carrier is moving in inertial space, the velocity disturbance in the inertial space will be generated. The disturbance is usually simulated by the sinusoidal disturbance generated by the angular vibrating table. In simulation, this sinusoidal disturbance is equivalent to the "total disturbances", which is added to the voltage input. The sinusoidal voltage disturbance with the amplitude of $1 \mathrm{~V}$ and frequency of $1.6 \mathrm{~Hz}$ is added to the system, as shown in the following figure.

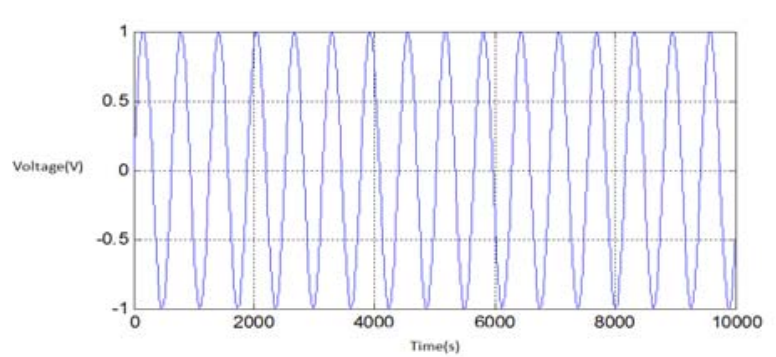

FIGURE VI. SINE VOLTAGE DISTURBANCE

Because of the friction torque between shafts, mass unbalance moment, winding torque and so on, there are disturbing torque single direction change in airborne photoelectric stabilized platform control system. Added voltage disturbance varies linearly with time $\mathrm{w}(\mathrm{k})=0.0008 \times \mathrm{k}+1$ to simulate the "total disturbance" of these torques, $\mathrm{k}$ changes with time, every 0.001 seconds increased 1 . In the simulation time of 10 seconds, the disturbance voltage increased from $1 \mathrm{~V}$ to $9 \mathrm{~V}$, as shown in the following show.

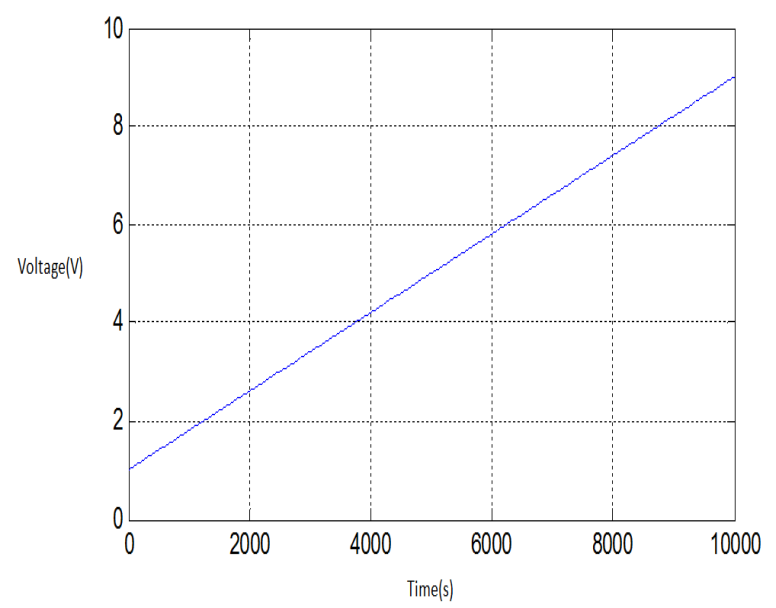

FIGURE VII. LINEAR VOLTAGE DISTURBANCE

\section{Simulation Methods and Results}

In order to study the performance characteristics of ADRC based on BP neural network, this paper uses MATLAB to simulate the ADRC based on BP neural network in airborne photoelectric stabilized platform.

According to the method shown in Figure 3, first select a set of coefficients $\left[3 \omega, 3 \omega^{2}, \omega^{3}\right] \times 2$ as the base value, including $\omega=400$, through repeated iteration to acquire a set of coefficients of $\left[\begin{array}{llll}450 & 120000 & 18000000\end{array}\right] \times 2$ as BP neural network output coefficients, the output values of the sigmoid function $O_{k 1}^{\text {out }} 、 O_{k 2}^{\text {out }} 、 O_{k 3}^{\text {out }}$, multiplied by 900,240000 and 18000000 respectively, and get $\left[\beta_{1}, \beta_{2}, \beta_{3}\right]$ as ESO three parameters.

There are 2 parameters $\mathrm{k}_{1}, \mathrm{k}_{2}$ of NLSEF control law need to be adjusted. We also used a method similar to ESO parameters tuning method to adjust the parameters of them. Inputs of the BP neural network are the system input, the 
system output, the feedback error and the bias value 1 , and the outputs are $\mathrm{k}_{1}$ and $\mathrm{k}_{2}$.

In order to verify the disturbance isolation effect of ADRC based on BP neural network, the nonlinear ADRC is adopted as control.

The output amplitude contrast is shown in the figure below. Fig.(a) is the output of the ADRC. Fig.(b) is the output of the ADRC based on BP neural network. The blue line is the system input value, and the red line is the system output value.

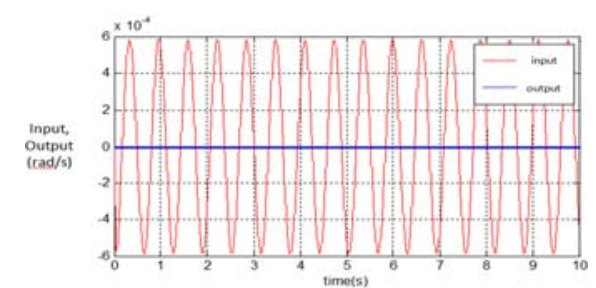

(a) ADRC without adding BP neural network

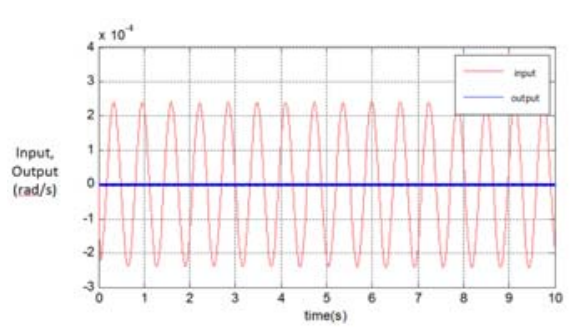

(a) ADRC based on BP neural network

FIGURE VIII. STABILIZAING ACCURACY CURVES WITH PLATFORM SINE DISTURBANCE(1)

Amplitude of Fig.(a) is about $6 \times 10-4 \mathrm{rad} / \mathrm{s}$, amplitude of Fig.(B) is about $2.4 \times 10-4 \mathrm{rad} / \mathrm{s}$, It can be seen that ADRC based on the BP neural network improved 1.5 times of sinusoidal disturbance isolation compared with traditional ADRC.

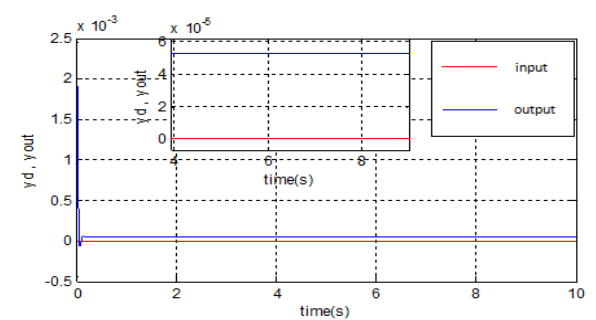

(a) ADRC without adding BP neural network

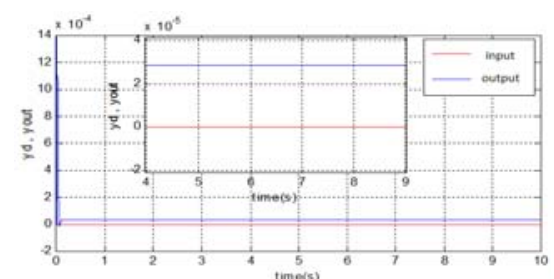

(a) ADRC based on BP neural network

FIGURE IX. STABILIZING ACCURACY CURVES WITH PLATFORM SINE DISTURBANCE(2)
Peak value of Fig.(a) is about $2.1 \times 10^{-3} \mathrm{rad} / \mathrm{s}$, steady-state value of Fig.(a) is about $5 \times 10^{-5} \mathrm{rad} / \mathrm{s}$. Peak value of Fig.(b) is about $1.4 \times 10^{-3} \mathrm{rad} / \mathrm{s}$, steady-state value of Fig.(b) is about $3 \times$ $10^{-5} \mathrm{rad} / \mathrm{s}$, It can be seen that ADRC based on the BP neural network improved $67 \%$ of torque disturbance isolation compared with traditional ADRC.

\section{CONCLUSION}

In this paper, the ADRC based on BP neural network in airborne photoelectric stabilized platform is studied, the stabilizing loop and disturbance are analyzed and modeled, the parameters tuning method of ADRC based on BP neural network parameters are analysed, the method to obtain the ESO parameter initial value is proposed. This method can effectively improve the convergence of BP neural network of ADRC. The ADRC based on BP neural network has been compared with the traditional ADRC in the airborne photoelectric stabilized platform through Simulation and Analysis.

The simulation results show that the parameters tuning method of the ADRC based on BP neural network can improve Disturbance isolation of airborne photoelectric stabilized platform significantly. This method has greatly improved the control effect has important significance to improve the LOS stabilization accuracy of airborne photoelectric stabilized platform.

\section{REFERENCES}

[1] Liu Xiang. "Theoretical Study on the Application of Line Accelerometer in Platform Stabilization System[D]." Changchun:Changchun. Institute of Optics,Fine Mechanics and Physics Chinese Academy of Science, 2004

[2] Zhang Bao. "Critical Technology of Aerial Photoelectric Imaging Platform[J]." Optics and Precision Engineering, 2007,15(8): 1280 .

[3] Zhao Xiu-yuan,Zhang Xin-li,Geng Le."Study of Active Disturbance Rejection on Extend State Observer[J]." Journal of Liaoning Normal University, 2011(34), 262265

[4] Zheng Ying. "Active Disturbance Rejection Research on Position Servo System of a Certain Cluster Rocket Launcher[D]." Nanjing University Science\&Technology, 2015.

[5] Han Jing-qing."Active Disturbance Rejection Control Technique-the technique for estimating and compensating the uncertainties[M]." Beijing: National Defend Industy Press, 2008.

[6] Zhiqiang Gao, "Scaling and bandwidth parameterization based controller tuning" Proceedings of the American Control Conference", Evanston, IL: IEEE, 2003: 49894996.

[7] QI Xiao-hui , LI Jie , HAN Shuai-tao.Adaptive Active Disturbance Rejection Control and Its Simulation Based on BP Neural Network[J]. ACTA ARMAMENTARII, 2013, 32(6)776-782

[8] Duan Hui-da.Cascade Auto-Disturbance-Rejection Control for a Class of High Order Nonlinear Systems with Model Uncertainty[D]. Changchun:Jilin University, 2012

[9] Li Xian-tao. "The Method for Restraint of Destabilization of Airborne Photoelectric Stabilized Platform[D]. ", Changchun: Changchun Institute of Optics, Fine Mechanics and Physics Chinese Academy of Science, 2014. 
[10] J.M. Hilkert. A reduced-order disturbance observer applied to inertially stabilized Line-of-Sight control [J]. Proc. Of SPIE, 2011 